\title{
Paleoclimatological perspective on the hydrometeorology of the Mekong Basin
}

\section{Räsänen T.A., Lehr C., Mellin I., Ward P.J. and Kummu M.}

This online supplement provides the spectral analysis results that were used to support the continuous wavelet transform (CWT) analyses. Fig. S1 shows the spectrums of basin averaged PDSI of the Stung Treng Catchment area (PDSI ${ }_{S T}$ ) and discharge at Stung Treng from the period 1910-2005 in frequency and time domains. Fig. S2 shows the spectrums of basin averaged PDSI of the Mekong (PDSI ${ }_{M}$ ) for the periods 1300-1557, 1575-1680, 1680-1780, 1780-1900, and 1900-2005 in frequency and time domains. The results in Fig. S1 and Fig. S2 are analysed together with the respective CWT analyses on PDSI $\mathrm{M}_{\text {, }}$ $\mathrm{PDSI}_{\mathrm{ST}}$ and discharge in the main paper.

A) PDSIst 1910-2005

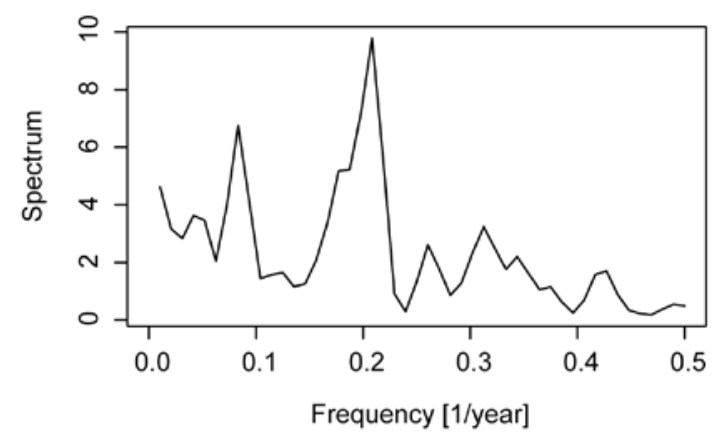

C) Discharge 1910-2005

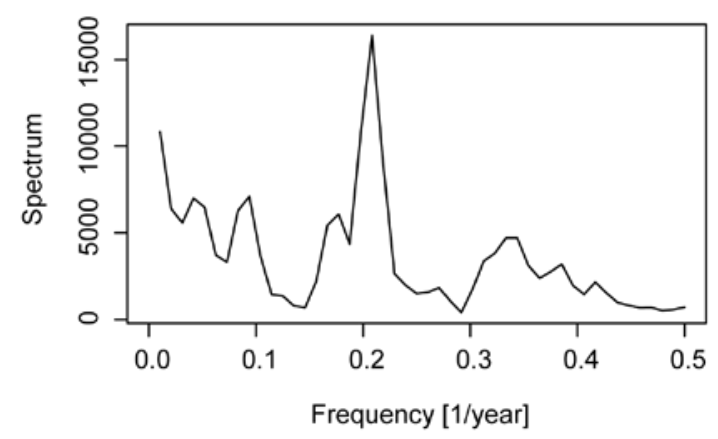

B) PDSIst 1910-2005

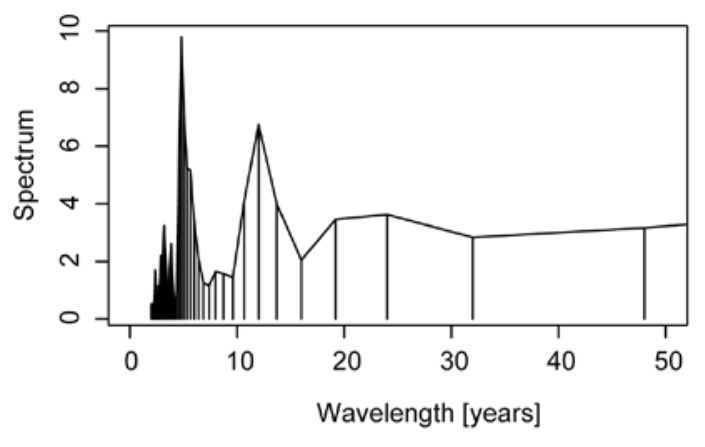

D) Discharge 1910-2005

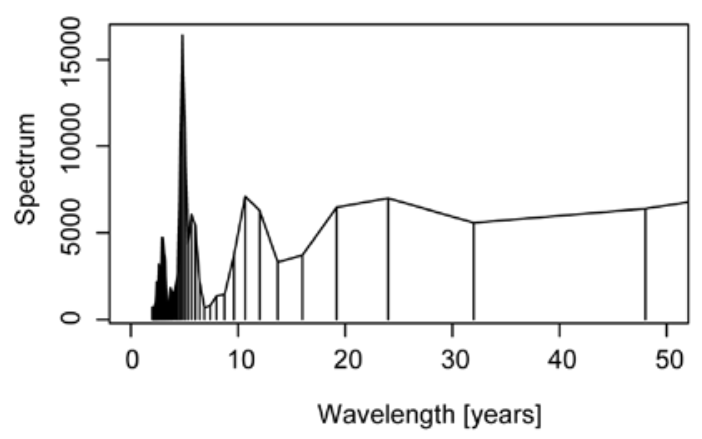

Fig. S1. Spectrums of basin averaged PDSI of the A,B) Stung Treng Catchment area (PDSIST) and C,D) discharge at Stung Treng from the period 1910-2005 in frequency and time domains. 
A. $1300-1575$

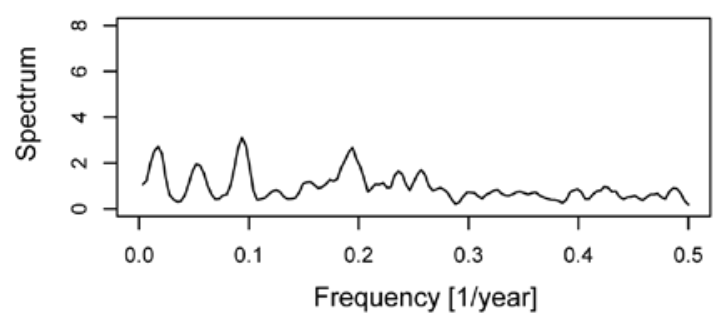

C. $1575-1680$

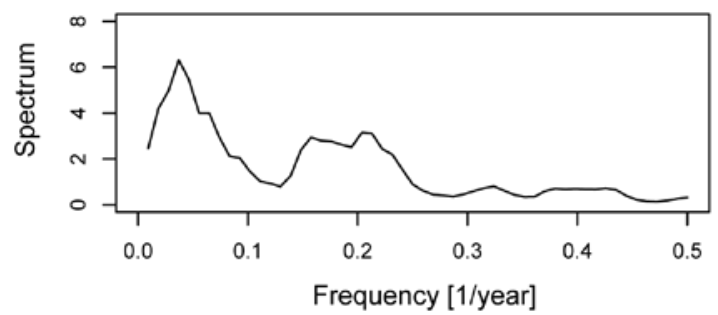

E. $1680-1780$

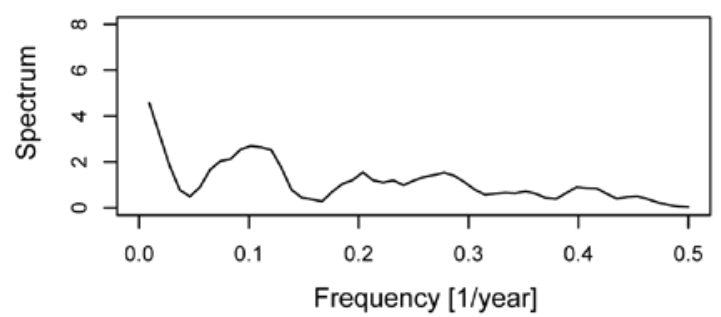

G. $1780-1900$

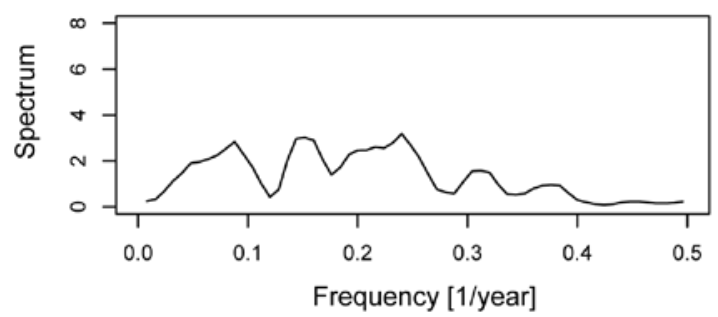

I. $1900-2005$

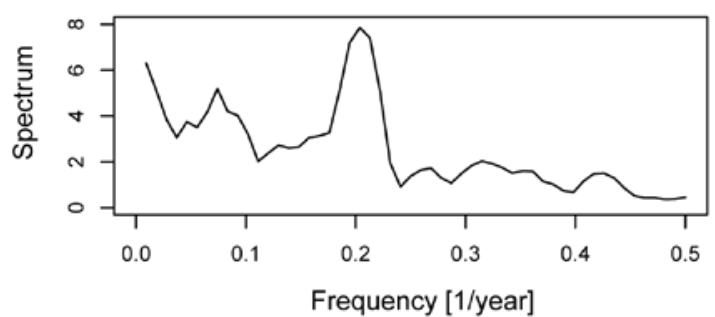

B. $1300-1575$

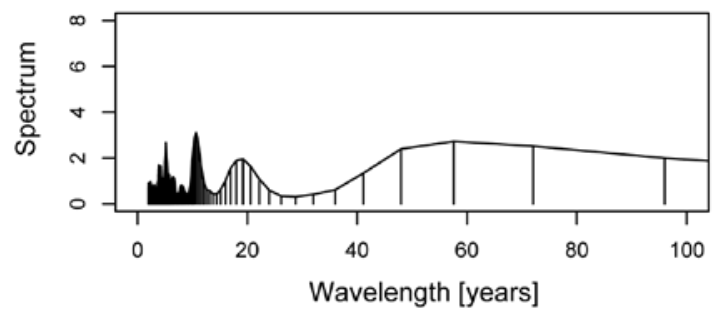

D. $1575-1680$

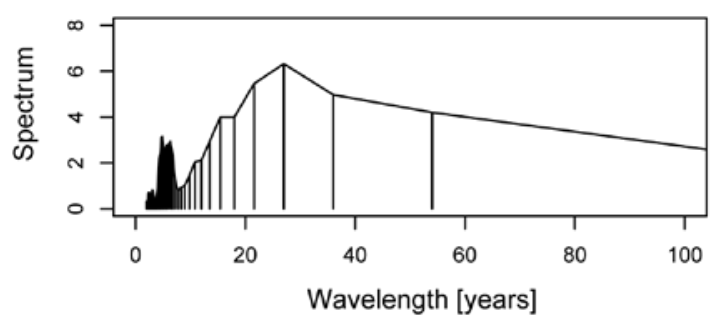

F. $1680-1780$

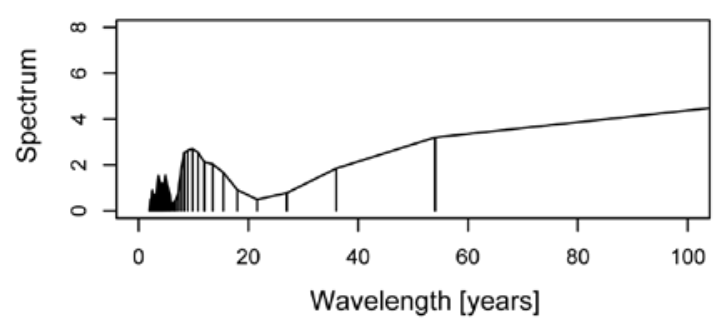

H. $1780-1900$

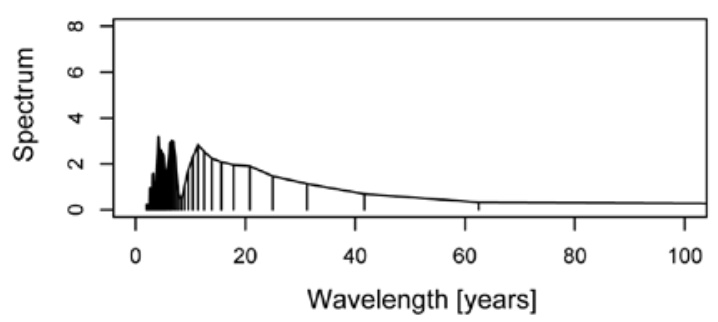

J. $1900-2005$

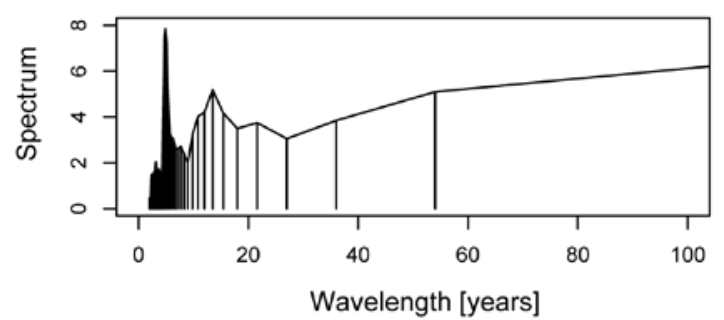

Fig. S2. Spectrums of basin averaged PDSI of the Mekong (PDSIM) from periods A,B) 1300-1557, B,C) 1575-1680, E,F) 1680-1780, G,H) 1780-1900 and I,J) 1900-2005 in frequency and time domains. 